\title{
The Future of MicroRNAs in Liver Cancer
}

\section{Drake G. LeBrun ${ }^{1}$, Xianjun $\mathrm{Yu}^{2}$ and $\mathrm{Min}^{\mathrm{Li}^{1 *}}$}

${ }^{1}$ The Vivian L. Smith Department of Neurosurgery, The University of Texas Health Science Center at Houston, Medical School, Houston, Texas 77030, USA ${ }^{2}$ Department of Pancreas \& Hepatobiliary Surgery, Shanghai Cancer Center, Department of Oncology, Shanghai Medical College, Fudan University, Shanghai 200032, China

\section{Keywords: MicroRNA; Liver cancer}

Liver cancer is a devastating disease that lacks effective therapeutic strategies. It is characterized by significant proliferative ability, high metastatic potential and high mortality. Moreover, it lacks validated diagnostic or therapeutic biomarkers. That is where microRNAs (miRNAs) come into focus. MiRNAs are small, non-coding RNA molecules with broad regulatory functions, controlling approximately $30 \%$ of the coding genes within the human genome [1]. The relatively recent discovery of microRNA (miRNA) deregulation in a number of human cancers has fueled an enormous surge in research on the potentially pivotal role of miRNAs in regulating tumorigenesis. Those studies have subsequently led to significant progress in identifying specific miRNAs as key oncogenes and tumor suppressors and have brought up the critical question: "What are the diagnostic and therapeutic impacts of miRNAs in liver cancer?" Emerging results suggest that miRNA expression profiles may be used to accurately diagnose liver cancer and provide specific prognoses for individual liver cancer patients. Moreover, targeting key oncogenic or tumor suppressive miRNAs is a possible treatment modality that has shown promising results in preclinical studies and merits further research.

Several studies have shown a differential miRNA expression profile in liver cancer tissues. Many of these studies have focused on elucidating the relationship between liver cancer and non-liver cancer tissues in general, while others have focused on comparing miRNA expression levels in normal liver tissue with those in specific liver cancer subtypes such as virally-associated malignancies, cancers of cirrhotic origin and cancers associated with hepatocellular stem cells (HSCs).

Out of these studies has arisen a relatively consistent miRNA expression profile that characterizes liver cancer from other tumors and normal liver tissues. Among the most frequently upregulated oncogenic miRNAs in liver cancer are miRNA-21, miRNA-221/222 and the miRNA-17-92 cluster. Conversely, miRNA-122, miRNA199a and the let-7 miRNA family are some of the most frequently downregulated tumor suppressive miRNAs in liver cancer. Several potential target genes of these miRNAs have been proposed, including Ras, PTEN, Cyclin G1, and mTOR [2-6]. Almost all of the proposed target genes are associated with hallmark cellular processes that characterize liver cancer cells: apoptotic resistance, increased cell growth and proliferation and enhanced invasiveness. These miRNA expression patterns should be of particular interest to clinicians, who in the future might be able to diagnose and classify liver tumors based on their miRNA profiles. In fact, tumor diagnosis and classification based on miRNA expression have been shown to be more accurate than mRNA-based profiling and miRNA profiling has further been shown to be an accurate predictor of liver cancer prognosis [7]. Clinically, this suggests that miRNA profiling may serve as a novel diagnostic and prognostic tool in liver cancer.

MiRNAs can also serve as therapeutic targets. Certain upregulated oncogenic miRNAs such as miRNA-21, miRNA-221 and miRNA-222 in liver cancer tissue have been specifically knocked down with anti-
miRNA oligonucleotides, antago-miRNAs, antisense molecules, ribozymes and DNAzymes. For example, a recent in vitro study found that downregulation of miRNA-221 and miRNA-222 in liver cancer cells by antago-miRNAs led to decreased cell growth [8]. On the other hand, downregulated tumor suppressive miRNAs can be restored through miRNA replacement therapies like miRNA mimics and viral vectormediated miRNA-overexpression. Kota et al. [9] showed that this is a viable strategy by increasing miRNA-26a levels using a miRNA-26a expressing adenovirus vector in a mouse model. This inhibited cell proliferation, increased tumor-specific apoptosis and enhanced protection from disease. Taken together, these two complementary strategies represent a promising avenue of therapeutic exploration, particularly in regard to providing personalized therapy for patients with liver cancer.

Despite the promise shown by emerging studies involving miRNAs in liver cancer, there are several limitations and roadblocks that have yet to be overcome in order for miRNA-based therapies to become viable and realistic at the bedside. First and foremost, miRNA-based therapies will only be useful if they are developed in tandem with effective delivery modalities. In order to distribute tumor suppressor miRNAs or antisense molecules to the liver, both systemic and local delivery methods need to be developed. Although some progress has been made in improving delivery systems, including the development of liposomal nanoparticles, lipid encapsulation methods and miRNA sponges, further work needs to be done to optimize these delivery modalities [10,11]. Moreover, some miRNAs have been observed to control over 100 target mRNAs and many target genes are yet unknown. Given the broad and incompletely defined activities of many miRNAs within a cell, manipulating miRNA expression levels may lead to unintended changes in key cellular processes. Additional work needs to be done to clarify and validate the potential cellular activities following miRNA expression changes.

MiRNAs represent an exciting research topic that will elucidate the molecular and cellular mechanisms of cancer progression and contribute to a promising avenue of liver cancer therapy. Due to their broad oncogenic and tumor suppressive properties, miRNAs can be mimicked or repressed to change the course of liver tumorigenesis. Moreover, miRNA profiling represents a promising diagnostic and therapeutic modality in a devastating cancer that lacks reliable diagnostic

*Corresponding author: Min Li, PhD, The Vivian L. Smith Department of Neurosurgery, The University of Texas Health Science Center at Houston, Medical School, 6431 Fannin Street, MSB 3.000, Houston, TX 77030, USA, Tel: (713) 5006491; E-mail: Min.Li@uth.tmc.edu

Received December 16, 2011; Accepted December 17, 2011; Published December 20, 2011

Citation: LeBrun DG, Yu X, Li M (2011) The Future of MicroRNAs in Liver Cancer J Liver 1:e101. doi:10.4172/2167-0889.1000e101

Copyright: (c) 2011 LeBrun DG, et al. This is an open-access article distributed under the terms of the Creative Commons Attribution License, which permits unrestricted use, distribution, and reproduction in any medium, provided the original author and source are credited. 
and prognostic biomarkers. By increasing our understanding of the roles of key aberrantly expressed miRNAs in liver cancer pathogenesis, we may be able to develop new strategies that enable earlier diagnosis and improved prognosis, enhance existing therapies, and improve patient outcomes.

Continued research on miRNA expression profiles and functions in liver cancer may help turn the tide in the quest to provide personalized and effective therapies for this destructive disease. Open access journals of the OMICS publishing group like the Journal of Liver will disseminate research developments about miRNAs and other promising topics to the scientific community free of charge, accelerate the process of scientific collaboration and quickly transform research revelations at the bench into real therapies at the bedside. Other special features of the OMICS publishing group journals include the digital book, audio version-enhanced feature of the website, language translation, social networking, 21 day rapid review process, team of 5,000 editors and 10,000 reviewers, one million readers, publication within 7 days of acceptance and quality and quick editorial, review processing.

\section{References}

1. Lewis BP, Burge CB, Bartel DP (2005) Conserved seed pairing, often flanked by adenosines, indicates that thousands of human genes are microRNA targets. Cell 120: 15-20.

2. Li W, Lebrun DG, Li M (2011) The expression and functions of microRNAs in pancreatic adenocarcinoma and hepatocellular carcinoma. Chin J Cancer 30 540-550.

3. Johnson CD, Esquela-Kerscher A, Stefani G, Byrom M, Kelnar K, et al. (2007) The let-7 microRNA represses cell proliferation pathways in human cells. Cancer Res 67: 7713-7722.

4. Olive $\mathrm{V}$, Jiang I, He L (2010) mir-17-92, a cluster of miRNAs in the midst of the cancer network. Int J Biochem Cell Biol 42: 1348-1354.

5. Gramantieri L, Ferracin M, Fornari F, Veronese A, Sabbiono S, et al. (2007) Cyclin G1 is a target of miR-122a, a microRNA frequently down-regulated in human hepatocellular carcinoma. Cancer Res 67: 6092-6099.

6. Fornari F, Milazzo M, Chieco P, Negrini M, Calin GA, et al. (2010) MiR -199a-3p regulates $\mathrm{mTOR}$ and $\mathrm{c}$-met to influence the doxorubicin sensitivity of human hepatocarcinoma cells. Cancer Res 70: 5184- 5193.

7. Lu J, Getz G, Miska EA, Alvarez-Saavedra E, Lamb J, et al. (2005) MicroRNA expression profiles classify human cancers. Nature 435: 834-838.

8. Pineau P, Volinia S, McJunkin K, Marchio A, Battiston C, et al. (2010) MiR-221 overexpression contributes to liver tumorigenesis. Proc Natl Acad Sci U S A 107: 264-269.

9. Kota J, Chivukula RR, O'Donnell KA, Wentzel EA, Montgomery CL, et al (2009) Therapeutic microRNA delivery suppresses tumorigenesis in a murine liver cancer model. Cell 137: 1005-1017.

10. Aigner A (2007) Nonviral in vivo delivery of therapeutic small interfering RNAs. Curr Opin Mol Ther 9: 345-352.

11. Ebert MS, Neilson JR, Sharp PA (2007) MicroRNA sponges: competitive inhibitors of small RNAs in mammalian cells. Nat Methods 4: 721-726. 